\title{
Exception to the Rule: Genomic Characterization of Naturally Occurring Unusual Vibrio cholerae Strains with a Single Chromosome
}

\author{
Gary Xie, ${ }^{1}$ Shannon L. Johnson, ${ }^{1}$ Karen W. Davenport, ${ }^{1}$ Mathumathi Rajavel, ${ }^{2}$ \\ Torsten Waldminghaus, ${ }^{3}$ John C. Detter, ${ }^{1}$ Patrick S. Chain, ${ }^{1}$ and Shanmuga Sozhamannan ${ }^{4,5}$ \\ ${ }^{1}$ Los Alamos National Laboratory, Biosciences Division, Genome Science, Los Alamos, NM 87545, USA \\ ${ }^{2}$ School of Computer, Mathematical and Natural Sciences, Morgan State University, Baltimore, MD 21251, USA \\ ${ }^{3}$ LOEWE Centre for Synthetic Microbiology-SYNMIKRO, Philipps-Universität Marburg, Hans-Meerwein-Str. 6, \\ 35032 Marburg, Germany \\ ${ }^{4}$ Tauri Group, LLC, Alexandria, VA 22310, USA \\ ${ }^{5}$ Defense Biological Product Assurance Office, 110 Thomas Johnson Drive, Frederick, MD 21702, USA
}

Correspondence should be addressed to Shanmuga Sozhamannan; shanmuga.sozhamannan.ctr@mail.mil

Received 21 February 2017; Revised 15 June 2017; Accepted 22 June 2017; Published 29 August 2017

Academic Editor: Graziano Pesole

Copyright @ 2017 Gary Xie et al. This is an open access article distributed under the Creative Commons Attribution License, which permits unrestricted use, distribution, and reproduction in any medium, provided the original work is properly cited.

\begin{abstract}
The genetic make-up of most bacteria is encoded in a single chromosome while about $10 \%$ have more than one chromosome. Among these, Vibrio cholerae, with two chromosomes, has served as a model system to study various aspects of chromosome maintenance, mainly replication, and faithful partitioning of multipartite genomes. Here, we describe the genomic characterization of strains that are an exception to the two chromosome rules: naturally occurring singlechromosome $V$. cholerae. Whole genome sequence analyses of NSCV1 and NSCV2 (natural single-chromosome vibrio) revealed that the Chr1 and Chr2 fusion junctions contain prophages, IS elements, and direct repeats, in addition to large-scale chromosomal rearrangements such as inversions, insertions, and long tandem repeats elsewhere in the chromosome compared to prototypical two chromosome $V$. cholerae genomes. Many of the known cholera virulence factors are absent. The two origins of replication and associated genes are generally intact with synonymous mutations in some genes, as are $\operatorname{rec} A$ and mismatch repair (MMR) genes dam, $m u t H$, and $m u t L$; MutS function is probably impaired in NSCV2. These strains are ideal tools for studying mechanistic aspects of maintenance of chromosomes with multiple origins and other rearrangements and the biological, functional, and evolutionary significance of multipartite genome architecture in general.
\end{abstract}

\section{Introduction}

The genetic endowment of the majority of bacteria ( 90\%) is inherited in a single chromosome of their respective genomes [1]. An exception to this rule is the presence of two chromosomes in $V$. cholerae, the causative agent of cholera disease $[2,3]$. In fact, all tested genera/species in the family Vibrionaceae possess two chromosomes [4]. The two V. cholerae chromosomes (Chr1 and $\mathrm{Chr} 2$ ) are of unequal sizes $(\sim 2.96 \mathrm{Mb}$ and $\sim 1.07 \mathrm{Mb}$, resp.). Most of our knowledge on the control of chromosome maintenance of multipartite genomes is derived from studies on
V. cholerae [5-7]. It was postulated that $\mathrm{Chr} 2$ is derived from plasmid based on its plasmid-like origin and evolved into a secondary chromosome by acquiring additional layers of regulation for its replication [3, 8]. Although Chr1 encodes the majority of the housekeeping genes and is considered as the main chromosome, Chr2 also harbors essential genes beside many genes with unknown functions [9-11]. The genes on Chr1 and Chr2 are differentially expressed in specific niches. For example, when the bacterium was grown midexponentially in rabbit ileal loops, it showed expression of many more genes of $\mathrm{Chr} 2$ than those expressed in aerobically grown cells in rich 
medium and harvested at the midexponential phase [12]. Majority of these are probably important niche-specific genes and hence expressed preferentially in ileal loop. The results were similar when the bacteria were collected from stools of cholera patients [13].

Chr1 replication follows the traditional E. coli paradigm in that the replication origin, oril, contains multiple DnaA boxes where DnaA binds to initiate DNA replication [14-16]. A V. cholerae Chr1 minireplicon can replicate in $E$. coli without the need for $V$. cholerae-transacting factors but merely using E. coli proteins such as DnaA. Similarly, $V$. cholerae oril could functionally substitute $E$. coli replication origin oriC $[11,14,16]$.

The $V$. cholerae ori2 resembles those of low-copynumber plasmids such as $\mathrm{P} 1$ and $\mathrm{F}$ in that it contains an array of repeats (iterons) where Chr2-specific initiator protein, RctB, binds to unwind the DNA for ori2 firing $[14,17]$. In $V$. cholerae, Chr1 initiates at the onset of the replication period while initiation of $\mathrm{Chr} 2$ is delayed and occurs only when $2 / 3$ of the Chr1 replication has already been completed. Because Chr2 is 1/3 the size of Chr1, both chromosomes terminate their replication roughly at the same time $[18,19]$. It was found recently that a site, termed crtS (Chr2 replication triggering site), present on Chr1 triggers ori2 initiation when it is replicated [20,21].

The two chromosomes of $V$. cholerae are longitudinally arranged in the cell [22]. While Chr1 appears to be spread along the entire longitudinal axis of the cell, $\mathrm{Chr} 2$ is restricted to the younger half of the cell. In newborn cells, Chr1 extends from the old pole to the new pole and $\mathrm{Chr} 2$ extends from midcell to the new pole [22]. The differential positioning of the chromosomes within the cell is accompanied by a distinct segregation choreography owing to chromosome-specific $\mathrm{ParAB} /$ parS-based segregation systems. [22-24]. One of the last steps in chromosome segregation before cell division involves the resolution of dimeric chromosomes that are frequently produced by homologous recombination between sister chromatids following DNA damage [25]. In V. cholerae, dimers of Chr1 and Chr2, are resolved by the action of the same machinery, XerC and XerD site-specific recombinases at the dif sites (dif1 and dif2), located in the ter regions of Chr1 and Chr2, respectively [26]. While the ter regions of both chromosomes replicate at the same time point within the cell cycle, Chrl sister termini are held together at midcell much longer than Chr2 sister termini [22]. The MatP/matS system was found to impede separation of Chrl sister termini and restrict movement of Chr2 sister termini to allow processing by FtsK right before cell division [27]. In addition, a nucleoid-associated, FtsZ-binding protein termed SImA has been shown to be required for blocking septal ring assembly. $\operatorname{SlmA}$ is a DNA-associated division inhibitor that is directly involved in preventing $\mathrm{Z}$ ring assembly on portions of the membrane surrounding the nucleoid [28].

$\mathrm{Chr} 2$ is indispensable for viability of the cell since elimination of Chr2 is lethal due to multiple "suicidal" toxinantitoxin systems encoded by Chr2 [29-31]. V. cholerae with single chromosomes has been created by genetic engineering to fuse the two natural chromosomes [32]. Chromosomal fusions in $V$. cholerae were also isolated as suppressor mutations for a deletion of the dam (DNA adenine methyl transferase) gene [33]. Dam is essential for replication of Chr2 because the initiator protein RctB binds to the target sites in ori2 only when they are methylated $[14,16,32]$. The only way to replicate Chr2 without a functional ori2, that is, unmethylated ori2, was a Chr1 and Chr2 chromosomal fusion that permits replication of the entire chromosome from ori1 [33]. In dam-suppressor mutants, Chrl and Chr2, fusions had occurred either by homologous recombination between IS elements or site-specific recombination between dif sites. Interestingly, fusion occurred preferentially in the terminus regions of the chromosomes [33]. Recently, it was found that chromosomal fusion in $V$. cholerae can also occur as a suppressor of $\Delta c r t S$ mutations [20].

Until recently, evidence for a naturally occurring Vibrio with fused chromosomes was missing. In an attempt to assess the genomic diversity of non-O1/non-O139 $\mathrm{V}$. cholerae, a whole genome mapping strategy was applied on a welldefined, geographically, and temporally diverse strain collection, the Sakazaki serogroup type strains [34]. In that study, the whole genome map data on 91 of the 206 serogroup type strains supported the hypothesis that $V$. cholerae has an unprecedented genetic and genomic structural diversity with very few clonal complexes. Fortuitously, chromosomal fusions in two unusual strains that possess a single chromosome instead of the two chromosomes usually found in $V$. cholerae were discovered which was further confirmed by pulse field gel electrophoresis (PFGE) [35].

Earlier, we reported the whole genome sequencing and generation of a gapless single-contig sequence of the two unusual single-chromosome strains, 1154-74 (serogroup O49) and 10432-62 (serogroup O27), hereafter referred to as NSCV1 and NSCV2, respectively [36]. In the current paper, we report further genome sequence analyses of NSCV1 and NSCV2. We delineate the Chr1 and Chr2 fusion junctions, other structural anomalies such as indels, inversions and duplications, salient features of their gene content and the origins of replication, and their potential activity. Furthermore, we analyze the genes involved in replication of the multiple origins in the same chromosome. Thus, the primary focus of this paper is to lay the foundation for future studies on functional and mechanistic aspects of chromosome and structural maintenance in these unusual $V$. cholerae strains.

\section{Materials and Methods}

2.1. Bacterial Strains and Growth Conditions. V. cholerae 1154-74 is a strain isolated in India in 1974 from a diarrheal sample, and it belongs to the $\mathrm{O} 49$ serogroup. $V$. cholerae 10432-62 is a strain isolated in the Philippines in 1962 from a diarrheal sample, and it belongs to the O27 serogroup. These strains are referred to as NSCV1 and NSCV2, respectively, in this manuscript. The corresponding Los Alamos National Labs (LANL) Sequencing Center designations are VAAO49 and VABO27, and the old locus tags in the Gen Bank entries carry these designations. The bacterial strains were grown in LB medium at $37^{\circ} \mathrm{C}$ using normal standard laboratory protocols. Genomic DNAs for sequencing 
were extracted using Qiagen genomic DNA extraction kits (Qiagen Inc.).

2.2. Whole Genome Sequencing Method. The draft genomes of Vibrio cholerae strains NSCV1 (1154-74) and NSCV2 (10432-62) were generated at the LANL Genome Science Group using a combination of Illumina [37] and 454 technologies [38]. For each genome, we constructed and sequenced an Illumina GAiiX shotgun library, a 454 Titanium standard library and a paired end 454 library, and a Pacific Biosciences RS long read library (P4-C2 chemistry). The 454 Titanium standard data and the 454 paired end data were assembled together with Newbler, version 2.3-PreRelease-6/30/2009. The Newbler consensus sequences were computationally shredded into $2 \mathrm{~kb}$ overlapping fake reads (shreds). Illumina sequencing data was assembled with VELVET, version 1.0.13 [39], and the consensus sequence was computationally shredded into $1.5 \mathrm{~kb}$ overlapping fake reads (shreds). We integrated the 454 Newbler consensus shreds, the Illumina VELVET consensus shreds, the PacBio subreads, and the read pairs in the 454 paired end library using parallel phrap, version SPS - 4.24 (High Performance Software, LLC). Illumina data were used to correct potential base errors and increase consensus quality using the software Polisher developed at Joint Genome Institute (JGI) [40]. Possible misassemblies were corrected using gapResolution [41], or Dupfinisher [42]. Completed genome assemblies were compared to optical maps to ensure consensus. Each final assembly consisted of a single ca $4.1 \mathrm{Mb}$ chromosome.

2.3. Genome Annotation. Assembled genomes were annotated using a modified version of the IGS Annotation Engine (released by the University of Maryland, Institute for Genome Sciences at the School of Medicine) on an Ergatis workflow manager. Annotated genomes are available in GenBank under accession numbers NSCV1-1154-74: NZ CP010811.1 and NSCV2-10432-62: NZ_CP010812.1. Post assembly genome sequence analysis was done using the CLC Genomic Workbench version 6.5.

Annotation of the assembled genome sequence was also carried out with genome annotation systems at LANL EDGE server https://bioedge.lanl.gov/ [43] and RAST server [44]. A combined gene prediction strategy was applied by means of the GLIMMER 2.0 system and the CRITICA program suite [45] along with post processing by the RBSfinder tool [46]. tRNA genes were identified with tRNAscan-SE [47]. The deduced proteins were functionally characterized by automated searches in public databases, including SWISS-PROT and TrEMBL [48], Pfam [49], TIGRFAM [50], InterPro [51], and KEGG [52]. Each gene was functionally classified by assigning clusters of orthologous group (COG) number and corresponding COG category [53].

2.4. Genomic Comparison. Comparative genome analyses of NSCV1 and NSCV2 to other V. cholerae strains MS6, O1 biovar El Tor str. N16961, and O139 MO10 were performed by using a set of tools available at LANL and RAST servers. Homology searches were conducted at the nucleotide and amino acid sequence level using BLAST [54]. To obtain a list of orthologs from bacteroidetes genomes, a perl script that determines bidirectional best hits was written. For example, genes $g$ and $h$ are considered orthologs if $h$ is the best BLASTP hit for $g$ and vice versa. $E$ values of $10^{-15}$ were acceptable. A gene is considered strain specific if it has no hits with an $E$ value of $10^{-5}$ or less. The genome comparisons at the nucleotide level were carried out with genome alignment tools, such as MUMmer2 [55], NUCmer [56], the Artemis Comparison Tool (ACT) [57], and WebAct (http://www. webact.org/WebACT/home) at Imperial College, London.

The MUMmer package [58] programs nucmer, repeat match, and exact_tandems were used for analysis of repeat regions. To identify long inexact genomic repeats, the nucmer program was used with the options - maxmatch and -nosimplify. The resulting dotplot was obtained using mummerplot. The repeat_match and exact_tandems programs were run with default arguments. Tandem repeats finder [59] and inverted repeats finder [60] were run with recommended default arguments to identify tandem and inverted repeats, respectively. Phage islands and putative genomic islands were identified using PHAST (PHAge Search Tool) [61] and Islandviewer [62], respectively. The circular genome diagram (Figure S3 available online at https://doi.org/10.1155/2017/8724304) was drawn using the DNAplotter [63]. Blast Ring Image Generator (BRIG) software was used to generate genome comparison views presented in Figure S2 [64]. Multiple genome alignment shown in Figure S1 was created using progressive MAUVE [65].

\section{Results and Discussion}

3.1. Whole Genome Sequencing of $V$. cholerae Strains NSCV1-(1154-74/VAA_O49 and NSCV2-(10432-62/VAB_ O27). Whole genome mapping (WGM) also known as optical mapping and PFGE data provided evidence of Chr1 and Chr2 fusions in V. cholerae strains NSCV1 (1154-74) and NSCV2 (10432-62) [35]. We performed whole genome sequencing of the two $V$. cholerae strains in order to understand the molecular mechanisms that might have led to Chr1 and Chr2 fusions in these strains [36]. We generated Roche $454(21 \times$ and $29 \times)$, Illumina $(2178 \times$ and $507 \times)$, and Pac Bio RS (350 $\times$ and $190 \times$ ) sequence data at the indicated coverages for NSCV1 and NSCV2, respectively, and used a hybrid assembly approach that resulted in a single-contig, gapless genome (Table S1). Further resolution of the ambiguous regions was carried out using Sanger-based primer walking according to Las Alamos National Labs (LANL) genome finishing pipeline yielding a final, finished, and closed single-contig sequence [66]. Consistent with the WGM results and in contrast to the previous genomic reports of $V$. cholerae and its close neighbors [2-4], these two sequences assembled into genomes consisting of a single chromosome of approximately the same size as the two expected chromosomes added together. All other genomic statistics for NSCV1 and NSCV2 appear to be highly similar to other $V$. cholerae genomes currently available in GenBank (Table S1). The genomes of NSCV1 and NSCV2 are comprised of a single circular chromosome of 3,928,357 


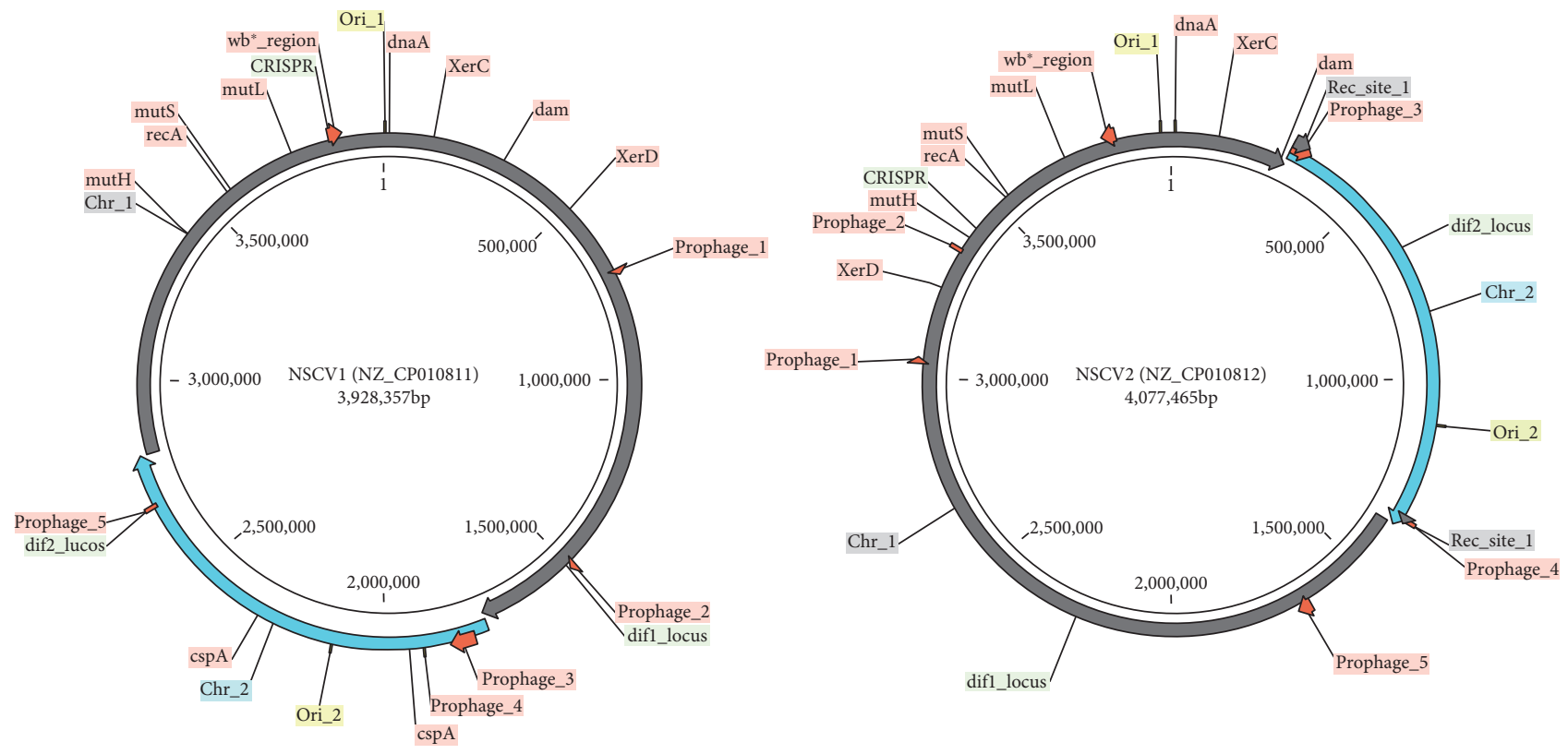

Figure 1: Circular genome maps of NSCV1 (1154-74_VAAO49) and NSCV2 (10432-62_VABO27). Fusion of Chr1 (dark grey) to Chr2 (blue) is shown in the circle at the respective locations. Various unique features such as prophages and the origins of replication- and replication-associated genes are indicated around the circles.

and 4,074,462 bp in length with an overall $\mathrm{G}+\mathrm{C}$ content of $47.73 \%$ and $47.66 \%$, respectively (Figure 1). Following the sequencing convention, the nucleotide position 1 was placed upstream of the dnaA gene (VAA_049_1 and VAB_027_1) encoding the chromosomal replication initiator protein. Chr2 portion of NSCV1 and NSCV2 spans from genome positions $1,720,246-2,772,395$ (1,052,150 bps) and 297,221$1,375,947$ (1,078,727 bps), respectively. All other NSCV1 and NSCV2 genome statistics are presented in Table S1.

3.2. Whole Genome Sequence Comparison of NSCV1 and NSCV2 to Other $V$. cholerae. DNA sequence of $V$. cholerae strain MS6 is the closest match to NSCV1 and NSCV2 chromosomes in public genome databases based on whole genome sequence homology [67]. MS6 is a $V$. cholerae O1 strain with a novel genetic background designated in Thailand-Myanmar and isolated from a stool sample of a diarrheal patient [68]. NSCV1 and NSCV2 genome sequences exhibit a high degree of synteny with MS6, N16961, MO10, and TSY216 as visualized in the Mauve alignment despite genetic rearrangements such as insertions and inversions across the chromosome (Figure S1) [69]. A pairwise Megablast comparison of NSCV1 and NSCV2 genomes to four pathogenic V. cholerae (N16961, MO10, MS6, and TSY 216) was performed. At 85\%, identity $>88-94 \%$ is shared between NSCV1 and NSCV2 compared to N16961, MS6, or MO10 genomes and 74\% and $80 \%$ is shared between NSCV1 and NSCV2 compared to TSY216, respectively. The unique regions varied from 5 to $10 \%$ (Table S2).

To further delineate the differences between NSCV1 and NSCV2 against all other genomes, the locations and gene content of the unique regions were determined (Table S3) and displayed as BRIG view (Figure S2). In the whole genome comparison of NSCV1 and NSCV2 to $V$. cholerae strains MS6, N16961, and TSY216, large regions ( $10 \mathrm{~kb}$ or more) missing in comparator strains are highlighted in the outer circle of the BRIG view (Figure S2). As expected, many features (e.g., prophages) that are unique to NSCV1 and NSCV2 are absent in the pathogenic strains and similarly, many of the known virulence regions (e.g., CTX and VPI) that are present in pathogenic $V$. cholerae such as N16961 are absent in NSCV1 and NSCV2. In Chr2 segment, the super integron region is present in NSCV1 and NSCV2 with many subtle indels/variations.

3.3. Comparison of Genomic Content of NSCV1 and NSCV2 to Pathogenic V. cholerae. Further analysis of the genomic content of NSCV1 and NSCV2 was performed by comparing the genome annotations. We were interested to see if there are any differences in the genetic content that might explain the mechanism of genomic fusion. Overall, the number of CDS encoded by NSCV1 and NSCV2 is very similar to other $V$. cholerae (Table S1). NSCV1 and NSCV2 have 2759 CDS in common with all other genomes compared here. Comparison of the genomes of NSCV1 and NSCV2 with MS6, N16961, and MO10 (an epidemic V. cholerae strain belonging to the O139 serogroup) revealed that the four organisms have approximately $3188 / 3259$ genes in common, depending on whether NSCV1 or NSCV2 is used as the query, respectively (Figure 2). There are 173/304 CDSs unique to NSCV1 and NSCV2, respectively.

3.4. Genomic Islands, Prophages, and Other Mobile Genetic Elements. In the NSCV1 and NSCV2 chromosomes, 49 and 20 genomic islands amounting to $677,625 \mathrm{bps}$ and 205 , 247 bps or $16.32 \%$ and $4.94 \%$ of the entire genome, respectively, were detected. Clustered regularly, interspaced short 


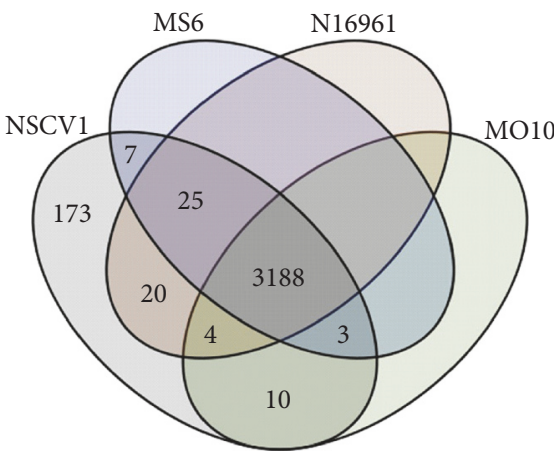

(a)

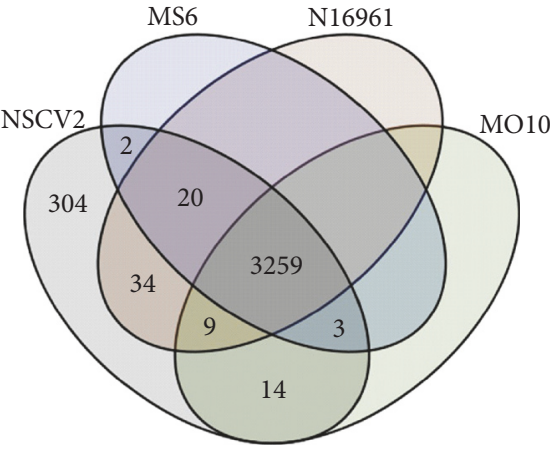

(b)

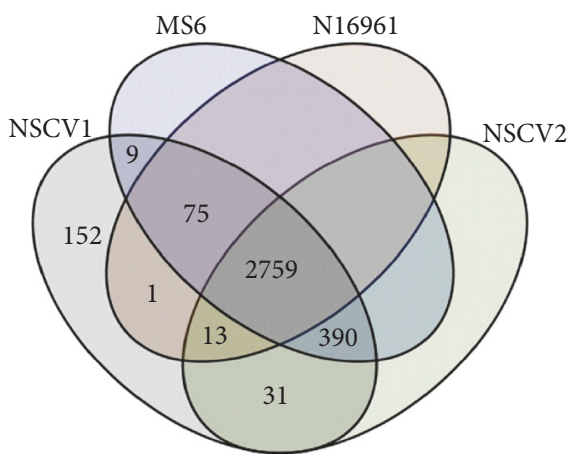

(c)

FIGURE 2: Comparison of the genomic content of NSCV1 (a), NSCV2 (b), and NSCV1 and NSCV2 (c) to various other genomes. Venn diagram showing the number of NSCV1 or NSCV2 predicted CDS with significant homology $\left(1 \mathrm{e}^{-5}\right)$ with the predicted products of the near neighbors: V. cholerae MS6, N16961 (serogroup O1 biovar El Tor), and O139 MO10 (serogroup O139). The number outside the circles (173 or 304) represents the number of NSCV1 or NSCV2 CDS that does not have significant homologs in the three strains compared. Conserved genes among them were defined by whole-genome pairwise sequence comparisons using the sequence-based comparison tool in RAST.

palindromic repeat (CRISPR) element is a widely found defense mechanism of prokaryotes against entry of foreign DNA including plasmids and phages [70]. In the NSCV1 and NSCV2 chromosomes, one CRISPR element was located at $3,606,088-3,606,203 \mathrm{bps}$ and $3,572,464-3,572,579 \mathrm{bps}$ in NSCV1 and NSCV2 genomes, respectively. The NSCV1 and NSCV2 genomes contained 58 and 55 mobile genetic elements, respectively, that encode phage integrases, transposases, and site-specific recombinase (Figure S3), compared to 46 mobile element genes in $V$. cholerae MS6. NSCV1 has 5 prophages located at (1) 685,729-702,622; (2) 1,458,739$1,476,484$; (3) $1,756,187-1,818,403$; (4) 1,879,405-1,883,642; and (5) 2,643,793-2,650,802. Prophages 3 and 5 are at the immediate boundary region and inside of the $\mathrm{Chr} 2$ insertion locus. Only prophage 3 at the $5^{\prime}$ end of $\mathrm{Chr} 2$ insertion appears to be intact and encodes 56 CDS. NSCV2 has 5 prophages located at (1) $3,113,341-3,130,219$; (2) $3,416,918-$ $3,428,588$; (3) 299,327-349,041; (4) 1,359,430-1,369,981; and (5) $1,676,903$ to $1,714,350$. Only prophage 5 is intact whereas the other four are defective or incomplete. Prophages 3 and 4 are at the immediate flanking region of the Chr2 insertion junction (Figure 1).

3.5. Virulence and Antimicrobial Resistance Genes. V. cholerae strains NSCV1 and NSCV2 were isolated from patients exhibiting atypical cholera symptoms [34]. It is of interest to see if these strains carry any of the usual $V$. cholerae virulence factors and if not, what other virulence factors or toxins might be present that would explain the diarrheal symptoms. The major virulence factor of $V$. cholerae, the cholera toxin encoded by $\operatorname{ct} x A B$ genes, is not found in NSCV1; however, other toxin genes ace and zot and RTX toxin cluster can be found, in addition to toxRS genes. The toxin genes are absent in NSCV2. NSCV1 and NSCV2 each has 57 genes encoding putative resistance to antibiotics and toxic compounds such as colicin V, bacteriocin, cobalt-zinc-cadmium, copper homeostasis, fluoroquinolones, and multidrug resistance efflux pumps.

3.6. O-Antigen Regions. NSCV1 and NSCV2 belong to serogroups $\mathrm{O} 49$ and O27, respectively, and as seen in other serogroups of $V$. cholerae, the $\mathrm{O}$-antigen biosynthesis genes are encoded in a cluster $\left(w b^{*}\right)$ on Chrl part of the respective genomes from 3,777,887-3,815,081 (CDS VAAO49_3428VAAO49_3463) and 3,888,708-3,920,841 (CDS VABO27 3582-VABO27_3611) of NSCV1 and NSCV2, respectively. Also, as seen in other $w b^{*}$ regions, the boundary regions of this cluster are highly conserved whereas the region between the conserved genes is highly divergent. More specifically, in the NSCV $1 w b^{*}$ region, 2 segments of 2974 bps and $6212 \mathrm{bps}$ at the left end and another segment of $5733 \mathrm{bps}$ at the right end, respectively, are $96 \%$ and $94 \%$ identical to those in the 


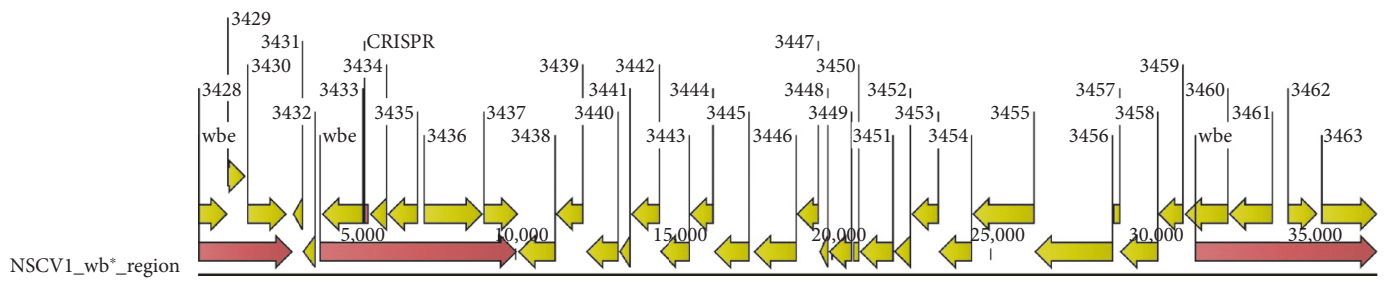

(a)

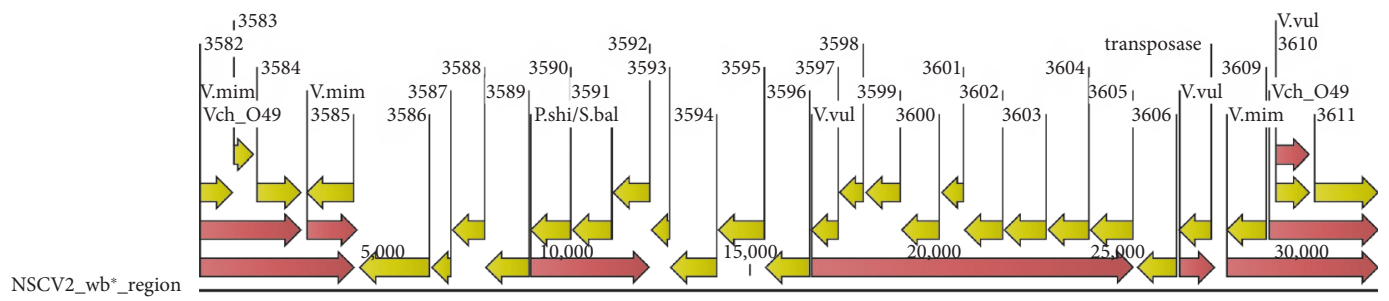

(b)

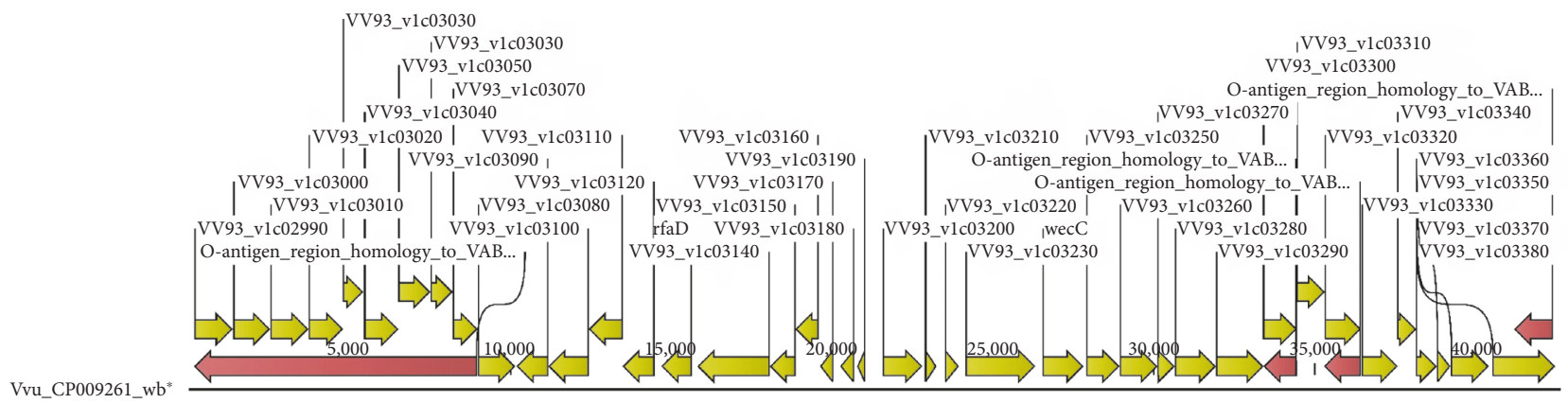

(c)

FIGURE 3: Genetic maps of O-antigen regions $\left(w b^{*}\right)$ of NSCV1 and NSCV2. The various genes and their orientations are indicated by the arrows. Homologous regions to other serogroups are indicated by the red arrows. The V. vulnificus $w b^{*}$ cluster that has extensive homology to NSCV2 $w b^{*}$ cluster is shown in (c).

$w b e$ region (O1 serogroup). A blast analysis of the $w b^{*}$ region of NSCV2 revealed segments that are similar to $V$. vulnificus (GenBank Accession \# CP009261) gene cluster (around $42 \mathrm{~kb}$ ) with 4 segments that are $>65-85 \%$ identical, ranging in length from $\sim 1.0 \mathrm{~kb}$ to $8.831 \mathrm{~kb}$ to the NSCV2 $w b^{*}$ cluster. In addition, identities to $V$. mimicus at $83-92 \%$ in segments of $4176 \mathrm{bps}, 2789 \mathrm{bps}$, and $1410 \mathrm{bps}$ and to Plesiomonas shigelloides and Shewanella baltica at $~ 80 \%$ (3281 bps), and to NSCV1 at $94-96 \%$ (4325, and 3007 bps) identities were also found as depicted in Figure 3.

3.7. Identification of Large Tandem Repeats and Inversion. A closer analysis of the WGMs (whole genome maps) of NSCV1 and NSCV2 strains revealed a large duplication in the general region of 1240 and $1506 \mathrm{~kb}$ on a reference genome (M66-2) coordinates based on optical restriction maps [35]. Experimental WGM data is further supported by in silico WGM generated from whole genome sequence data of NSCV1 and NSCV2 (Figure S4). Sequence read mapping results showed that there is a large duplication of $200 \mathrm{kbs}$ and $70 \mathrm{kbs}$ regions in NSCV1 and NSCV2, respectively, as evidenced by $>1 \times$, but $<2 \times$, depth of average coverage of the genome at $1,503,484$ to $1,718,861 \mathrm{bps}$ in NSCV1 and 2,221,105 to 2,300,924 bps in NSCV2 compared to that in the rest of the genome (Figure S5). Manual validation indicated that the $200 \mathrm{kbs}$ and $70 \mathrm{kbs}$ regions indeed are fragmental duplications, existing among subpopulation of NSCV1 and NSCV 2 cells, respectively. Both 1 copy and 2 copy versions of genome assembly have enough evidence supporting variations among population. These duplicated sequences spanned from positions $1,503,484$ to $1,718,861$ (unit 1) and from $1,718,862$ to $1,934,269 \mathrm{bp}$ (unit 2) in NSCV1 and from positions 2,221,105 to 2,300,924 (unit 1) and from 2,300,925 to 2,380,745 (unit 2) in NSCV2 in genome sequences with repeats included (Figure S6). The duplicated sequences span a total length of 430,785 bp and 159,640 bp on Chr1 region and encode 177 and 59 duplicated genes in NSCV1 and NSCV2, respectively. It should be noted that the two copies of repeat units are not identical. There is a $31 \mathrm{bp}$ indel between the two copies in NSCV1, which resides at the locus of VAA_049_1509, encoding exonuclease family protein. There are 5 single bp indels between the two copies in NSCV2. Although tandem repeats are present at different locations on NSCV1 and NSCV2, there is a $40 \mathrm{~kb}$ DNA segment that overlapped at the $5^{\prime}$ end of NSCV1 and NSCV2 repeats with $98 \%$ identities (Figure S7). However, the tandem duplications could not be verified by PCR of the junctions since the orientation of the two copies cannot be ascertained in the assembly. Hence, we have decided to keep the genome sequence with a single 
copy as the ref seq (GenBank submission) for all the analyses. This long duplication anomaly could not be unequivocally resolved with the existing sequence data. In addition, there is a large inversion from $1,477,189$ to $3,484,983$ bps in NSCV2 compared to MS6 chromosome 1 region from 612,067 to 2,541,958 bps (Figure S1b).

3.8. Mapping the Fusion Junctions and Possible Mechanism of Genome Fusion. Chromosomal fusions were observed previously in suppressor mutants resulting from a genetic screen [33]. These fusions were analyzed in detail and found to occur via two different mechanisms, either by homologous recombination at IS elements or by site-specific recombination at dif sites [32] or as transient chromosomal fusions in $\Delta c r t S$ suppressor mutants [20]. In this study, we analyzed the fusion sites of NSCV1 and NSCV2 in detail to see if any one of these mechanisms also led to chromosomal fusion in the natural single-chromosome isolates. The circular single chromosomes of NSCV1 and NSCV2 are shown in Figure 1, and along with N16961, Chr1- and Chr2concatenated circular maps are shown in Figure S2, and the genome sequence statistics are provided in Table S1. The insertion sites on Chr1 backbone (as an accepter) and Chr2 backbone (as a donor) are not the same for NSCV1 and NSCV2 (see below). Artemis Comparison Tool views (zoomed in view) of the fusion junctions are presented in Figures S8 and S9. Further description of the hypothetical events leading to the fusions is provided below.

3.9. Chr1 and Chr2 Fusion Junctions in V. cholerae Strain NSCV1. A closer examination of the Chr1-Chr2 insertion junction in NSCV1 to Chr1 and Chr2 sequences of MS6 indicates that multiple events probably occurred before the final NSCV1 was derived. In an NSCV1-like intermediate, on Chr1 between VAA049_1545 and VAA049_2500, there probably was an insertion of MS6_A0562- and MS6_A0272-like CDS (Chr2 CDS) (Figure 4 (top panel)). On Chr2, there was an insertion of a prophage to the right of MS6_A0272 (Figure 4 (top panel)). In the next step, Chr2 with the new prophage was inserted into Chr1 via the homologies between the two CDS (VAA049_1594 versus MS6_A0272 and VAA049_2432 versus MS6_A0562), and upon the resolution of the cointegrate, one copy each of the two homologous CDS along with intervening sequences was deleted (Figure 4 (bottom panel)). Thus, Chr2 in NSCV1 spans from $1,714,319$ to $2,772,395$ bps (including its flanking unique region, Figure 4 (bottom panel)) or from 1,757,711 to 2,722,145 bps (including boundary of Chr2 homologous region) (Figure 4 (bottom panel)) spanning NSCV1 CDS VAAO49_1594-2432. This recombination event likely occurred between MS6_1029 and MS6_1028 or between $1,168,144$ and $1,168,021$ bp in MS6-like Chr1 background and inserted from the breakpoint between MS6_A0272 and MS6_A0562 or between 303,999 and 446,488 bp on MS6like Chr2 background (Figure 4 (bottom panel)). There are 2 prophages ( 3 and 5 ) closer to the boundaries of Chr2 insertion locus. Flanking the $\mathrm{Chr} 2$ insertion sites, there are unique regions of $37,425 \mathrm{bps}$ and $50,250 \mathrm{bps}$ in length at $5^{\prime}$ end and $3^{\prime}$ end, respectively. The role of any of these unique regions in the recombination event or the precise mechanism of recombination cannot be ascertained with available whole genome sequence data in public databases.

3.10. Chr1 and Chr2 Fusion Junctions in V. cholerae Strain NSCV2. A closer examination of the insertion junctions indicates that an NSCV2-like intermediate probably possessed MS6_A0925- and MS6_A0924-like Chr2 CDS on Chrl flanked by 2 prophages (Figure 5 (top panel)). In that intermediate strain, Chr2 was inserted via recombination of the homologous segments (VAB027_307 versus MS6_A0924 and VAB027_1228 versus MS6_A0925). As a result, Chr2 spans from $337,822 \mathrm{bps}$ to $1,351,337 \mathrm{bps}$ (without its flanking prophage regions between CDS VABO27_307VABO27_1228) or from $297,221 \mathrm{bps}$ to $1,375,947 \mathrm{bps}$ (including prophage regions between CDS VABO27_275VABO27_1225). This recombination event likely occurred between 2,643,219 and 2,643,208 bp or between MS6_2336 and MS6_2335 on MS6 Chr1 backbone and inserted between MS6_A0924 and MS6_A0925 break points (852,904852,973 ) on MS6 Chr2 backbone (Figure 5 (bottom panel)). The insertion boundary is flanked by $245 \mathrm{bp}$ repeats located at 337,552 to $337,796 \mathrm{bp}$ and $1,351,093$ to $1,351,337 \mathrm{bps}$ with $96 \%$ identities, then flanked by 2 prophages. In the flanking regions of 2 prophages, there are $12 \mathrm{bp}$ identical repeats (caccgcagggtg) (297,210-297,221 bps and 1,375,947$1,375,958 \mathrm{bps})$. The $245 \mathrm{bps}$ repeat also overlaps $155 \mathrm{bps}$ with VABO27_1228 that encodes L-threonine 3-dehydrogenase.

As mentioned above for NSCV1, the role of any of these unique regions in the recombination event or the mechanism of recombination that resulted in chromosomal fusion cannot be ascertained with available $V$. cholerae whole genome sequence data. Since the immediate predecessor or recombination intermediate strains are not known, it is difficult to decipher the exact events that led to the NSCV1 and NSCV2. For example, the exact mechanism of recombination (site specific versus generalized recombination) or the precise recombination cross-over points cannot be predicted. It also appears that there is more than one event before the final NSCV1 and NSCV2 arose. The presence of prophages at the insertion junctions leads us to speculate that the Chr2 insertion events in NSCV1 and NSCV2 appear to be mediated by generalized homologous recombination via homologies provided by prophages or parts/genes homologous to prophage genes and mobile gene elements present in both Chr1 and Chr2 in the precursor strain.

3.11. Sequence Analysis of the Origins of Replication and Associated Genes. The ori1 and ori2 origins of replications in NSCV1 and NSCV2 chromosomes were identified by a homology to the Chr1 and Chr2 origins of MS6. The ori1 is colocalized with the genes $(r p m H, d n a A, d n a N, \operatorname{rec} F$, and $g y r A$ ) often found near the oriC in prokaryotic genomes, and origin of the location corresponded with GC nucleotide skew $(\mathrm{G}-\mathrm{C} / \mathrm{G}+\mathrm{C})$ analysis as illustrated in Figure S2 and Figure S3. Based on these data, we assigned base-pair 1 in an intergenic region located in the putative oril. Similarly, ori 2 was located based on sequence homology to the ori2 in other prototypical $V$. cholerae genomes. The genome 


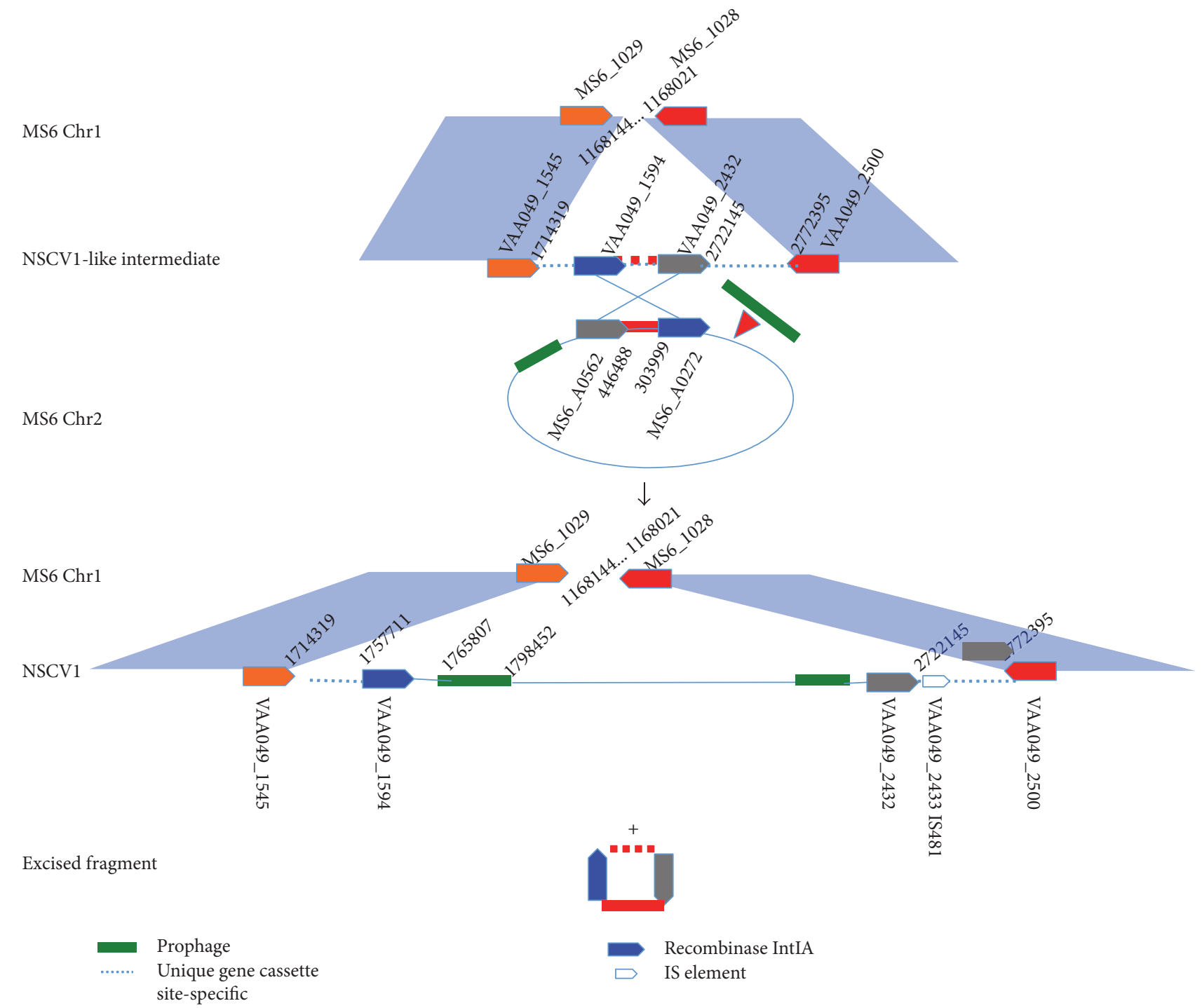

FIgURE 4: Putative recombination event that resulted in Chr1 and Chr2 fusions in NSCV1. Top panel: MS6 Chr1 CDS at the fusion junction and the same location in a putative NSCV1-like intermediate and the MS6 Chr2 circle and the codons at the fusion junction are depicted. The cross-over region between $\mathrm{Chr} 1$ and $\mathrm{Chr} 2$ is indicated by the long $\mathrm{X}$. A prophage insertion event to the right fusion junction prior or post to Chr 2 fusion is depicted by a green line. Bottom panel: The recombination products after fusion event are shown with NSCV 1 in the middle and an excision product that contains one copy of the cross-over genes with the intervening sequences at the bottom.

positions of the ori, their orientation, and genes within the ori are indicated in Table 1. A list of putative dif sites (chromosome dimer resolution sites) and their locations are provided in Table S4. A previous study had found chromosome fusions in $V$. cholerae as suppressors of impaired Chr2 replication [33]. As mentioned above, some of these fusions had occurred by site-specific recombination of the two dif sites of Chr1 and Chr2, respectively. The positions of the intact dif sites on the fused chromosomes in NSCV1 and NSCV2 support the notion that this was not the mechanism how fusion occurred in these strains (Table S4). It remains to be seen which of the two dif sites on NSCV1 and NSCV2 chromosomes is active and used for chromosome dimer resolution. This might depend on the molecular mechanism involved in positioning the respective XerCD recombinase at the dif site.
There is extensive genetic conservation in the oril and ori2 of NSCV1 (Figure 6) and NSCV2 (Figure 7) compared to a prototypical reference genome such as N16961. A closer look at the sequences of the origin regions including the genes within the origin of replication indicated that there is no significant indels between the respective origins compared to a reference genome such as N16961. However, a number of SNPs were found in the origins of replication. The nucleotide and amino acid changes at the origins of NSCV1 and NSCV2 are presented in Table S5. It remains to be analyzed experimentally if both origins on the fused chromosomes are active in replication.

3.12. Sequence Analyses of Replication Associated and Mismatch Repair Genes That May Be Potentially Involved in Single-Chromosome Maintenance. Earlier studies have 


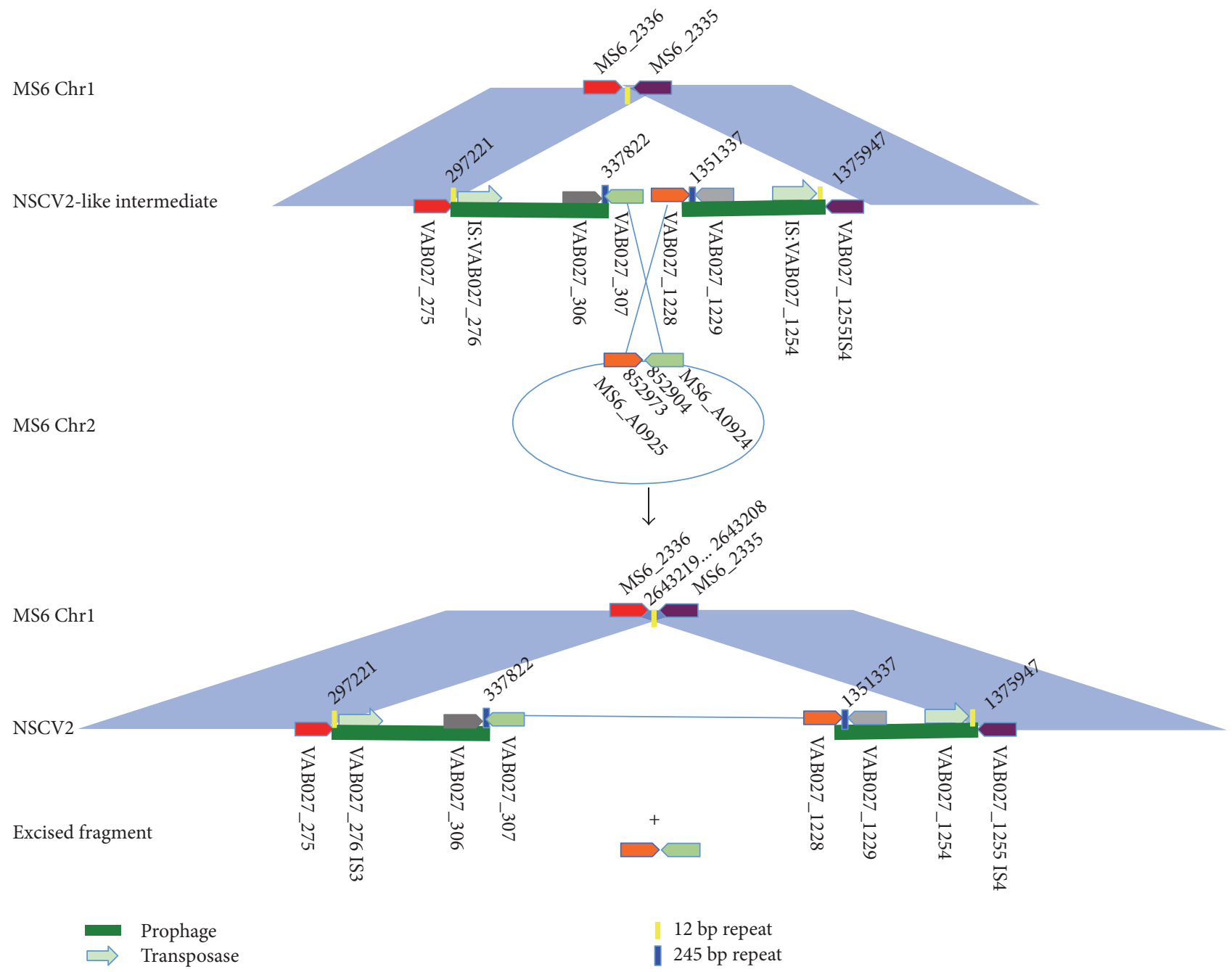

FIGURE 5: Putative recombination event that resulted in Chr1 and Chr2 fusions in NSCV2. Top panel: MS6 Chr1 CDS at the fusion junction and the same location in a putative NSCV2-like intermediate and the MS6 Chr2 circle and the codons at the fusion junction are depicted. The cross-over region between $\mathrm{Chr} 1$ and $\mathrm{Chr} 2$ is indicated by the long X. Bottom panel: The recombination products after fusion event are shown with NSCV2 in the middle and an excision product that contains one copy of the cross-over genes with the intervening sequences at the bottom.

indicated that the dam gene is essential for the viability of $V$. cholerae, and depletion of DNA adenine methyl transferase (Dam protein) leads to successful spontaneous fusion of the two chromosomes [33]. Hence, we examined the genetic status of DNA adenine methylase gene (dam) in natural single-chromosome $V$. cholerae, NSCV1, and NSCV2 and found the dam gene to be intact. We also inspected the status of RecA and the MMR genes since they have been implicated in maintenance of chromosomal rearrangements such as large tandem repeats, inversion, and fusion. The nucleotide and amino acid changes in various replication and MMR genes in NSCV1 and NSCV2 are presented in Table S6. RecA-mediated homologous recombination was probably involved in the fusion event, and the fact that the resolution of the single chromosome into 2 chromosomes has not been observed during normal growth conditions indicates that the RecA in NSCV1 and NSCV2 is nonfunctional or altered due to the presence of multiple SNPs, of which one leads to single-amino acid change in RecA protein (Y305C). Preliminary data indicate that NSCV1 and NSCV2 are probably recombination deficient since construction of recombinants via natural transformation has been unsuccessful. The MMR genes are generally intact except for mutS. The mutS gene is impaired, that is, deletion of amino acid residues $132-150$, in NSCV2. Among the other mismatch repair system genes, mutH and mutL have single-nucleotide polymorphisms that lead to amino acid changes in the respective proteins. Other replication-associated proteins such as DnaA, ParAB, and XerC have amino acid changes whereas XerD is intact (Table S6). Preliminary data indicate that the ori1 is active in both strains suggesting a functional DnaA protein. However, the effect of these protein alterations in ParAB, XerC, RecA, and MMR proteins on recombination, replication, and maintenance of chromosomal fusions and other large-scale genome rearrangements described above awaits more stringent functional studies including cloning and intra-/interspecific complementation and these studies are underway. 


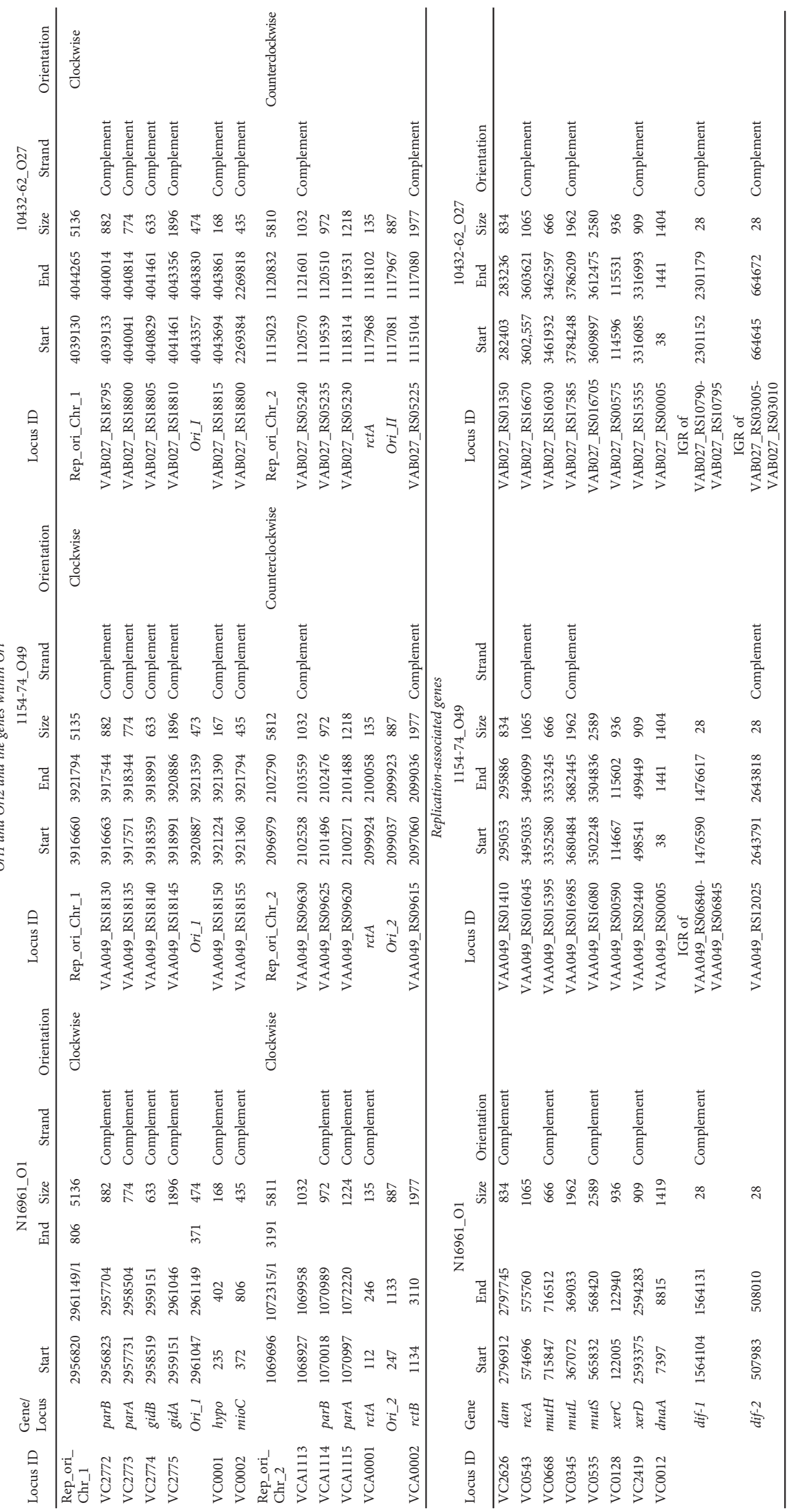




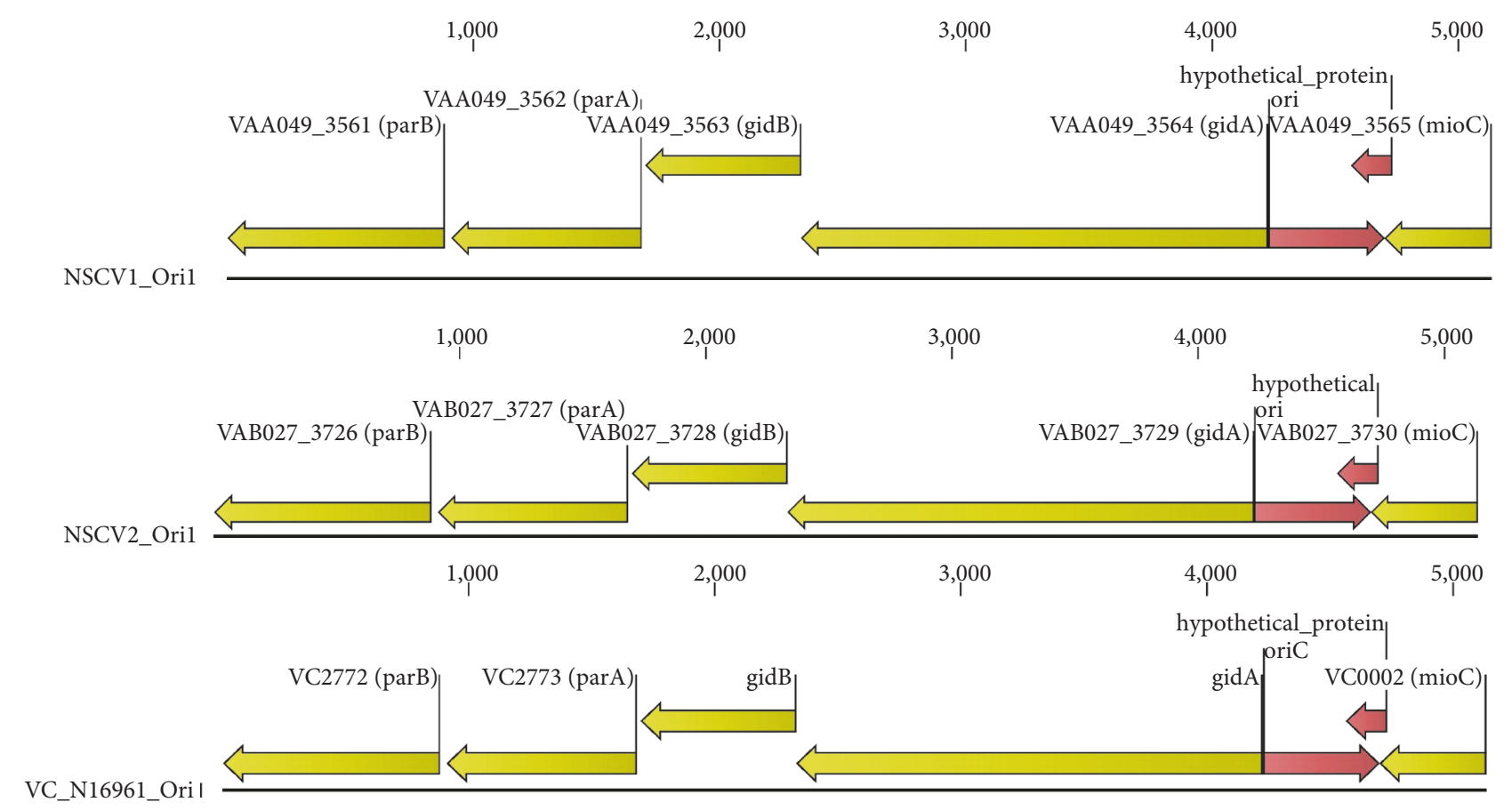

FIgURE 6: Genetic organization of ori1 of NSCV1 and NSCV2 in comparison to the respective ori in N16961. The old locus tags with known gene designations have been used to indicate the ORFs. The physical ori and unannotated ORFs are indicated by red arrows.

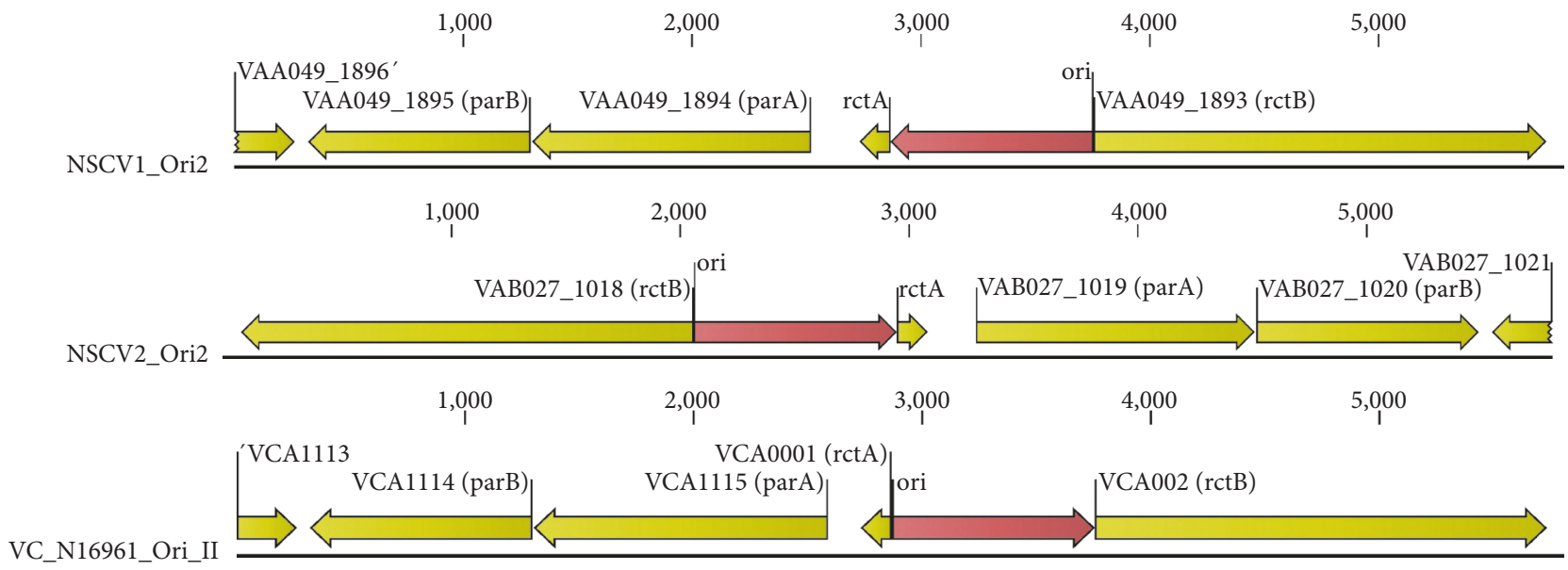

FIGURE 7: Genetic organization of ori2 of NSCV1 and NSCV2 in comparison to the respective ori in N16961. The old locus tags with known gene designations have been used to indicate the ORFs. The physical ori is indicated by a red arrow.

\section{Conclusions}

All species of the genus Vibrio known to date harbor two chromosomes. Here, we present an exception to this rule by describing the genomic architecture of two natural $V$. cholerae isolates with one fused chromosome. For many years, the quest to understand why and how Vibrios evolved their bipartite genomes remains enigmatic. The strains described here appeared to have taken an evolutionary path backwards and might be instrumental in future unraveling of longstanding questions on chromosome biology in Vibrios. One fascinating question to address is whether the replication of the fused chromosome is dominated by one of the subchromosomes or if they share the two replicons. If the latter is true then this would be the first example of a bacterial chromosome with two active replication origins. A tantalizing idea that has been proposed is that Chr2 is actually a "parasitic replicon." The concept of selfish replication origins has been established recently [71]. It could be that the $V$. cholerae strains described here are the result of a selfish replicon taking over the "host." Currently, studies are underway to begin to address these questions to unravel the mystery of a single chromosome with multiple replication origins. A second question that is worth exploring pertains to how the chromosomal fusions are maintained and if and how or under what conditions they 
ever revert into two separate chromosomes. Many of the genes involved in these functions have suffered changes. It remains to be seen if NSCV1 and NSCV2 strains are defective or functionally altered in any of these genes in order to maintain the chromosomal fusions and other structural alterations.

\section{Abbreviations \\ NSCV: Natural single-chromosome vibrio \\ WGM: Whole genome map (optical map) \\ WGS: Whole genome sequence \\ PFGE: Pulse field gel electrophoresis \\ LANL: Los Alamos National Labs \\ JGI: Joint Genome Institute \\ BRIG: Blast Ring Image Generator.}

\section{Additional Points}

Availability of Supporting Data. Whole genome sequences are available in GenBank under accession numbers NSCV1-1154-74: NZ_CP010811.1 and NSCV2-10432-62: NZ_CP010812.1.

\section{Conflicts of Interest}

The authors declare no conflict of interests.

\section{Acknowledgments}

This work was supported by a grant of the Deutsche Forschungsgemeinschaft (Grant no. WA 2713/4-1) to Torsten Waldminghaus. This article is approved by LANL for unlimited release (LA-UR-17-21600).

\section{References}

[1] A. A. Prozorov, "Additional chromosomes in bacteria: properties and origin," Mikrobiologiia, vol. 77, no. 4, pp. 437-447, 2008.

[2] M. Trucksis, J. Michalski, Y. K. Deng, and J. B. Kaper, “The Vibrio cholerae genome contains two unique circular chromosomes," Proceedings of the National Academy of Sciences of the United States of America, vol. 95, no. 24, pp. 14464-14469, 1998.

[3] J. F. Heidelberg, J. A. Eisen, W. C. Nelson et al., "DNA sequence of both chromosomes of the cholera pathogen Vibrio cholerae," Nature, vol. 406, no. 6795, pp. 477-483, 2000.

[4] K. Okada, T. Iida, K. Kita-Tsukamoto, and T. Honda, "Vibrios commonly possess two chromosomes," Journal of Bacteriology, vol. 187, no. 2, pp. 752-757, 2005.

[5] E. S. Egan, M. A. Fogel, and M. K. Waldor, "Divided genomes: negotiating the cell cycle in prokaryotes with multiple chromosomes," Molecular Microbiology, vol. 56, no. 5, pp. 1129-1138, 2005.

[6] J. K. Jha, J. H. Baek, T. Venkova-Canova, and D. K. Chattoraj, "Chromosome dynamics in multichromosome bacteria," Biochimica et Biophysica Acta, vol. 1819, no. 7, pp. 826-829, 2012.

[7] M. E. Val, A. Soler-Bistué, M. J. Bland, and D. Mazel, "Management of multipartite genomes: the Vibrio cholerae model," Current Opinion in Microbiology, vol. 22, pp. 120-126, 2014.
[8] T. Venkova-Canova and D. K. Chattoraj, "Transition from a plasmid to a chromosomal mode of replication entails additional regulators," Proceedings of the National Academy of Sciences of the United States of America, vol. 108, no. 15, pp. 6199-6204, 2011.

[9] D. E. Cameron, J. M. Urbach, and J. J. Mekalanos, “A defined transposon mutant library and its use in identifying motility genes in Vibrio cholerae," Proceedings of the National Academy of Sciences of the United States of America, vol. 105, no. 25, pp. 8736-8741, 2008.

[10] M. C. Chao, J. R. Pritchard, Y. J. Zhang et al., "High-resolution definition of the Vibrio cholerae essential gene set with hidden Markov model-based analyses of transposon-insertion sequencing data," Nucleic Acids Research, vol. 41, no. 19, pp. 9033-9048, 2013.

[11] H. D. Kamp, B. Patimalla-Dipali, D. W. Lazinski, F. WallaceGadsden, and A. Camilli, "Gene fitness landscapes of Vibrio cholerae at important stages of its life cycle," PLoS Pathogens, vol. 9, no. 12, article e1003800, 2013.

[12] Q. Xu, M. Dziejman, and J. J. Mekalanos, "Determination of the transcriptome of Vibrio cholerae during intraintestinal growth and midexponential phase in vitro," Proceedings of the National Academy of Sciences of the United States of America, vol. 100, no. 3, pp. 1286-1291, 2003.

[13] D. S. Merrell, S. M. Butler, F. Qadri et al., "Host-induced epidemic spread of the cholera bacterium," Nature, vol. 417, no. 6889, pp. 642-645, 2002.

[14] E. S. Egan and M. K. Waldor, "Distinct replication requirements for the two Vibrio cholerae chromosomes," Cell, vol. 114, no. 4, pp. 521-530, 2003.

[15] S. Duigou, K. G. Knudsen, O. Skovgaard, E. S. Egan, A. Løbner-Olesen, and M. K. Waldor, "Independent control of replication initiation of the two Vibrio cholerae chromosomes by DnaA and RctB," Journal of Bacteriology, vol. 188, no. 17, pp. 6419-6424, 2006.

[16] G. Demarre and D. K. Chattoraj, "DNA adenine methylation is required to replicate both Vibrio cholerae chromosomes once per cell cycle," PLoS Genetics, vol. 6, no. 5, article e1000939, 2010.

[17] S. Duigou, Y. Yamaichi, and M. K. Waldor, “ATP negatively regulates the initiator protein of Vibrio cholerae chromosome II replication," Proceedings of the National Academy of Sciences of the United States of America, vol. 105, no. 30, pp. 1057710582, 2008.

[18] T. Rasmussen, R. B. Jensen, and O. Skovgaard, "The two chromosomes of Vibrio cholerae are initiated at different time points in the cell cycle," The EMBO Journal, vol. 26, no. 13, pp. 3124-3131, 2007.

[19] C. Stokke, T. Waldminghaus, and K. Skarstad, "Replication patterns and organization of replication forks in Vibrio cholerae," Microbiology, vol. 157, Part 3, pp. 695-708, 2011.

[20] M. E. Val, M. Marbouty, F. de Lemos Martins et al., "A checkpoint control orchestrates the replication of the two chromosomes of Vibrio cholerae," Science Advances, vol. 2, no. 4, article e1501914, 2016.

[21] J. H. Baek and D. K. Chattoraj, "Chromosome I controls chromosome II replication in Vibrio cholerae," PLoS Genetics, vol. 10, no. 2, article e1004184, 2014.

[22] A. David, G. Demarre, L. Muresan, E. Paly, F. X. Barre, and C. Possoz, "The two cis-acting sites, parS1 and oriC1, 
contribute to the longitudinal organisation of Vibrio cholerae chromosome I," PLoS Genetics, vol. 10, no. 7, article e1004448, 2014.

[23] J. Livny, Y. Yamaichi, and M. K. Waldor, "Distribution of centromere-like parS sites in bacteria: insights from comparative genomics," Journal of Bacteriology, vol. 189, no. 23, pp. 8693-8703, 2007.

[24] Y. Yamaichi, M. A. Fogel, S. M. McLeod, M. P. Hui, and M. K. Waldor, "Distinct centromere-like parS sites on the two chromosomes of Vibrio spp," Journal of Bacteriology, vol. 189, no. 14, pp. 5314-5324, 2007.

[25] C. Lesterlin, F. X. Barre, and F. Cornet, "Genetic recombination and the cell cycle: what we have learned from chromosome dimers," Molecular Microbiology, vol. 54, no. 5, pp. 1151-1160, 2004.

[26] M. E. Val, S. P. Kennedy, M. El Karoui, L. Bonné, F. Chevalier, and F. X. Barre, "FtsK-dependent dimer resolution on multiple chromosomes in the pathogen Vibrio cholerae," PLoS Genetics, vol. 4, no. 9, article e1000201, 2008.

[27] G. Demarre, E. Galli, L. Muresan et al., "Differential management of the replication terminus regions of the two Vibrio cholerae chromosomes during cell division," PLoS Genetics, vol. 10, no. 9, article e1004557, 2014.

[28] T. G. Bernhardt and P. A. de Boer, "SlmA, a nucleoidassociated, FtsZ binding protein required for blocking septal ring assembly over chromosomes in E. coli," Molecular Cell, vol. 18, no. 5, pp. 555-564, 2005.

[29] J. Yuan, Y. Yamaichi, and M. K. Waldor, "The three vibrio cholerae chromosome II-encoded ParE toxins degrade chromosome I following loss of chromosome II," Journal of Bacteriology, vol. 193, no. 3, pp. 611-619, 2011.

[30] Y. Yamaichi, M. A. Fogel, and M. K. Waldor, "Par genes and the pathology of chromosome loss in Vibrio cholerae," Proceedings of the National Academy of Sciences of the United States of America, vol. 104, no. 2, pp. 630-635, 2007.

[31] N. Iqbal, A. M. Guérout, E. Krin, F. Le Roux, and D. Mazel, "Comprehensive functional analysis of the 18 Vibrio cholerae N16961 toxin-antitoxin systems substantiates their role in stabilizing the superintegron," Journal of Bacteriology, vol. 197, no. 13, pp. 2150-2159, 2015.

[32] M. E. Val, O. Skovgaard, M. Ducos-Galand, M. J. Bland, and D. Mazel, "Genome engineering in Vibrio cholerae: a feasible approach to address biological issues," PLoS Genetics, vol. 8, no. 1, article e1002472, 2012.

[33] M. E. Val, S. P. Kennedy, A. J. Soler-Bistué et al., "Fuse or die: how to survive the loss of dam in Vibrio cholerae," Molecular Microbiology, vol. 91, no. 4, pp. 665-678, 2014.

[34] T. Shimada, E. Arakawa, K. Itoh et al., "Extended serotyping scheme for Vibrio cholerae," Current Microbiology, vol. 28, no. 3, pp. 175-178, 1994.

[35] C. Chapman, M. Henry, K. A. Bishop-Lilly et al., "Scanning the landscape of genome architecture of non-O1 and non-O139 Vibrio cholerae by whole genome mapping reveals extensive population genetic diversity," PLoS One, vol. 10, no. 3, article e0120311, 2015.

[36] S. L. Johnson, A. Khiani, K. A. Bishop-Lilly et al., "Complete genome assemblies for two single-chromosome Vibrio cholerae isolates, strains 1154-74 (serogroup O49) and 10432-62 (serogroup O27)," Genome Announcements, vol. 3, no. 3, 2015.

[37] S. Bennett, "Solexa Ltd," Pharmacogenomics, vol. 5, no. 4, pp. 433-438, 2004.
[38] M. Margulies, M. Egholm, W. E. Altman et al., "Genome sequencing in microfabricated high-density picolitre reactors," Nature, vol. 437, no. 7057, pp. 376-380, 2005.

[39] D. R. Zerbino and E. Birney, "Velvet: algorithms for de novo short read assembly using de Bruijn graphs," Genome Research, vol. 18, no. 5, pp. 821-829, 2008.

[40] B. Foster, K. LaButti, S. Trong, C. Han, T. Brettin, and A. Lapidus, "POLISHER: A tool for using ultra short reads in genome sequence improvement," 2009, - Report Number: LBNL-2792E Poster https://pubarchive.lbl.gov/ islandora/object/ir\%3A153560.

[41] S. Trong, K. LaButti, B. Foster, C. Han, T. Brettin, and A. Lapidus, "Gap resolution: a software package for improving Newbler genome assemblies," in Sequencing, Finishing, Analysis in the Future Meeting, Santa Fe, NM, 2009.

[42] C. Han and P. Chain, "Finishing repeat regions automatically with Dupfinisher," in Proceedings of the 2006 International Conference on Bioinformatics \& Computational Biology, $\mathrm{H}$. R. Arabnia and H. Valafar, Eds., pp. 141-146, CSREA Press, Las Vegas, NV, USA, 2006.

[43] P. E. Li, C. C. Lo, J. J. Anderson et al., "Enabling the democratization of the genomics revolution with a fully integrated web-based bioinformatics platform," Nucleic Acids Research, vol. 45, no. 1, pp. 67-80, 2017.

[44] R. K. Aziz, D. Bartels, A. A. Best et al., "The RAST server: rapid annotations using subsystems technology," BMC Genomics, vol. 9, p. 75, 2008.

[45] A. C. McHardy, A. Goesmann, A. Pühler, and F. Meyer, "Development of joint application strategies for two microbial gene finders," Bioinformatics, vol. 20, no. 10, pp. 1622-1631, 2004.

[46] B. E. Suzek, M. D. Ermolaeva, M. Schreiber, and S. L. Salzberg, "A probabilistic method for identifying start codons in bacterial genomes," Bioinformatics, vol. 17, no. 12, pp. 1123-1130, 2001.

[47] T. M. Lowe and S. R. Eddy, "tRNAscan-SE: a program for improved detection of transfer RNA genes in genomic sequence," Nucleic Acids Research, vol. 25, no. 5, pp. 955964, 1997.

[48] B. Boeckmann, A. Bairoch, R. Apweiler et al., "The SWISSPROT protein knowledgebase and its supplement TrEMBL in 2003," Nucleic Acids Research, vol. 31, no. 1, pp. 365-370, 2003.

[49] A. Bateman, E. Birney, L. Cerruti et al., "The Pfam protein families database," Nucleic Acids Research, vol. 30, no. 1, pp. 276-280, 2002.

[50] D. H. Haft, J. D. Selengut, and O. White, "The TIGRFAMs database of protein families," Nucleic Acids Research, vol. 31, no. 1, pp. 371-373, 2003.

[51] N. J. Mulder, R. Apweiler, T. K. Attwood et al., "The InterPro database, 2003 brings increased coverage and new features," Nucleic Acids Research, vol. 31, no. 1, pp. 315318, 2003.

[52] M. Kanehisa and S. Goto, "KEGG: kyoto encyclopedia of genes and genomes," Nucleic Acids Research, vol. 28, no. 1, pp. 27-30, 2000.

[53] R. L. Tatusov, N. D. Fedorova, J. D. Jackson et al., "The COG database: an updated version includes eukaryotes," $B M C$ Bioinformatics, vol. 4, p. 41, 2003.

[54] S. Altschul, T. L. Madden, A. A. Schäffer et al., "Gapped BLAST and PSI-BLAST: a new generation of protein 
database search programs," Nucleic Acids Research, vol. 25, pp. 3389-3402, 1997.

[55] A. L. Delcher, A. Phillippy, J. Carlton, and S. L. Salzberg, "Fast algorithms for large-scale genome alignment and comparison," Nucleic Acids Research, vol. 30, no. 11, pp. 2478-2483, 2002.

[56] S. Kurtz, A. Phillippy, A. L. Delcher et al., "Versatile and open software for comparing large genomes," Genome Biology, vol. 5, no. 2, p. R12, 2004.

[57] T. J. Carver, K. M. Rutherford, M. Berriman, M. A. Rajandream, B. G. Barrell, and J. Parkhill, "ACT: the Artemis Comparison Tool," Bioinformatics, vol. 21, no. 16, pp. 3422-3423, 2005.

[58] S. Kurtz, A. Phillippy, A. L. Delcher et al., "Versatile and open software for comparing large genomes," Genome Biology, vol. 5, article R12, 2004.

[59] G. Benson, "Tandem repeats finder: a program to analyze DNA sequences," Nucleic Acids Research, vol. 27, pp. 573580, 1999.

[60] P. E. Warburton, J. Giordano, F. Cheung, Y. Gelfand, and G. Benson, "Inverted repeat structure of the human genome: the X-chromosome contains a preponderance of large, highly homologous inverted repeats that contain testes genes," Genome Research, vol. 14, pp. 1861-1869, 2004.

[61] Y. Zhou, Y. Liang, K. H. Lynch, J. J. Dennis, and D. S. Wishart, "PHAST: a fast phage search tool," Nucleic Acids Research, vol. 39, pp. W347-W352, 2011.

[62] B. K. Dhillon, M. R. Laird, J. A. Shay et al., "IslandViewer 3: more flexible, interactive genomic island discovery, visualization and analysis," Nucleic Acids Research, vol. 43, no. W1, pp. W104-W108, 2015.

[63] T. Carver, N. Thomson, A. Bleasby, M. Berriman, and J. Parkhill, "DNAPlotter: circular and linear interactive genome visualization," Bioinformatics, vol. 25, no. 1, pp. 119$120,2009$.

[64] N. F. Alikhan, N. K. Petty, N. L. Ben Zakour, and S. A. Beatson, "BLAST ring image generator (BRIG): simple prokaryote genome comparisons," BMC Genomics, vol. 12, p. 402, 2011.

[65] A. E. Darling, B. Mau, and N. T. Perna, "ProgressiveMauve: multiple genome alignment with gene gain, loss and rearrangement," PLoS One, vol. 5, no. 6, article e11147, 2010.

[66] P. S. Chain, D. V. Grafham, R. S. Fulton et al., "Genomics. Genome project standards in a new era of sequencing," Science, vol. 326, no. 5950, pp. 236-237, 2009.

[67] K. Okada, M. Na-Ubol, W. Natakuathung et al., "Comparative genomic characterization of a Thailand-Myanmar isolate, MS6, of Vibrio cholerae O1 El Tor, which is phylogenetically related to a "US Gulf Coast" clone," PLoS One, vol. 9, no. 6, article e98120, 2014.

[68] K. Okada, A. Roobthaisong, W. Swaddiwudhipong, S. Hamada, and S. Chantaroj, "Vibrio cholerae O1 isolate with novel genetic background, Thailand-Myanmar," Emerging Infectious Diseases, vol. 19, no. 6, pp. 1015-1017, 2013.

[69] A. C. Darling, B. Mau, F. R. Blattner, and N. T. Perna, "Mauve: multiple alignment of conserved genomic sequence with rearrangements," Genome Research, vol. 14, no. 7, pp. 1394-1403, 2004.
[70] R. Barrangou, C. Fremaux, H. Deveau et al., "CRISPR provides acquired resistance against viruses in prokaryotes," Science, vol. 315, no. 5819, pp. 1709-1712, 2007.

[71] M. Hawkins, S. Malla, M. J. Blythe, C. A. Nieduszynski, and T. Allers, "Accelerated growth in the absence of DNA replication origins,” Nature, vol. 503, no. 7477, pp. 544-547, 2013. 

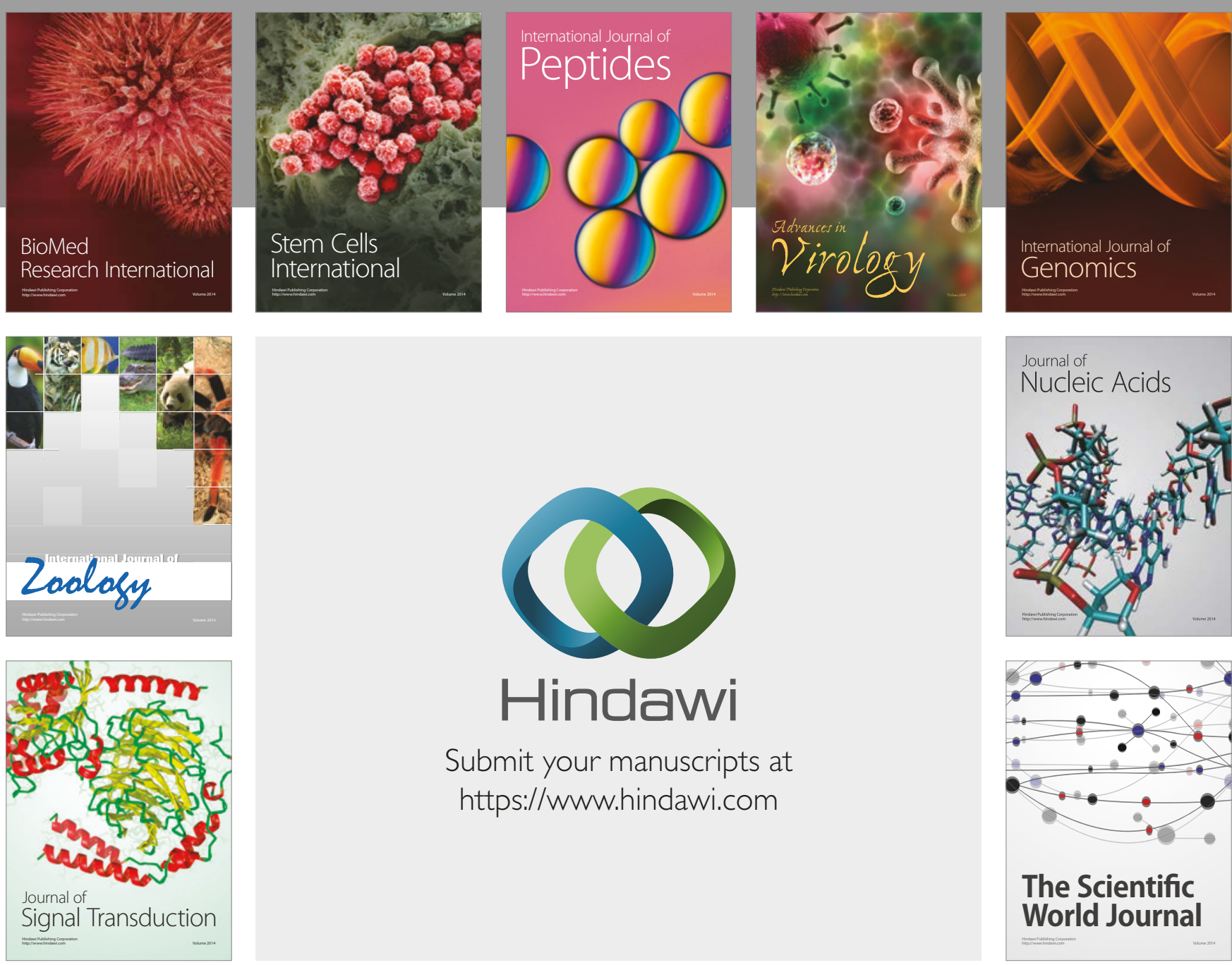

Submit your manuscripts at

https://www.hindawi.com
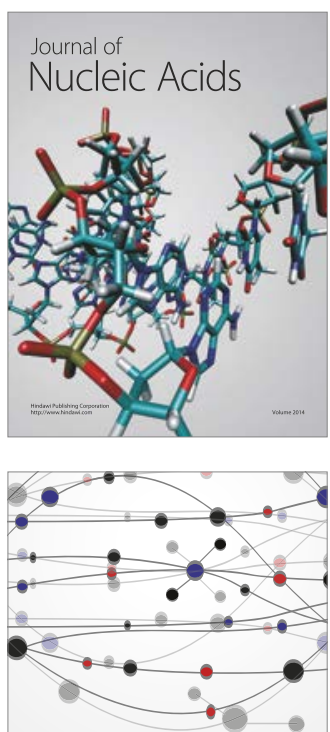

The Scientific World Journal

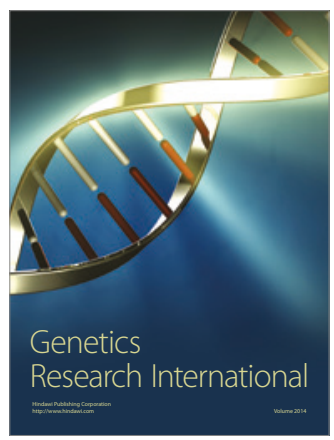

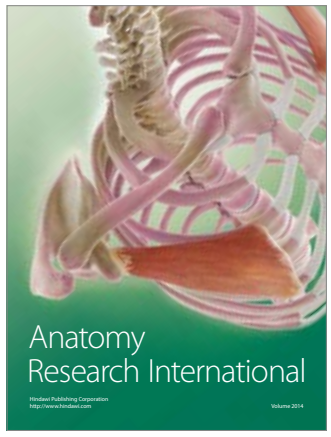

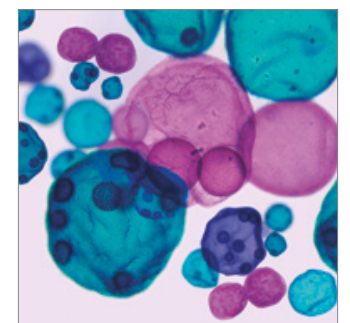

International Journal of Microbiology
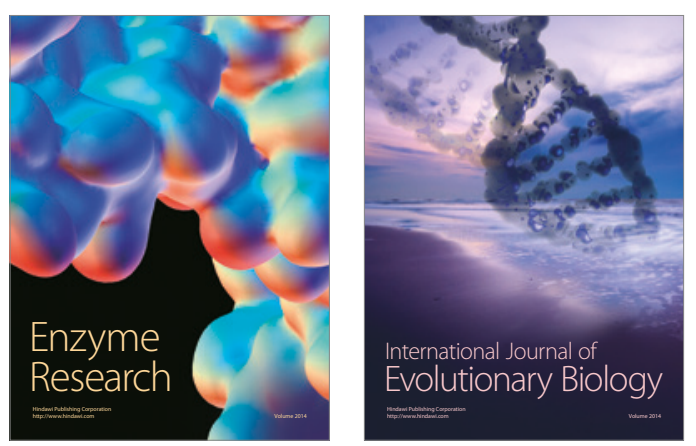
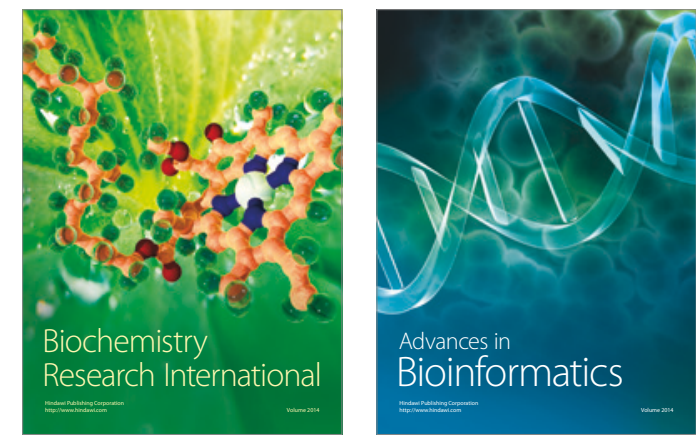

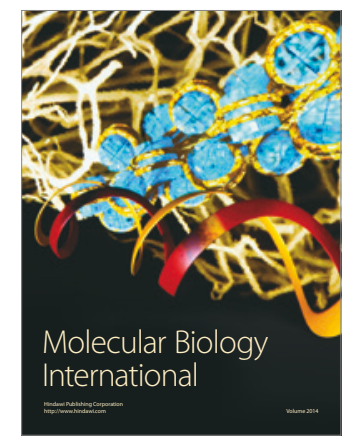

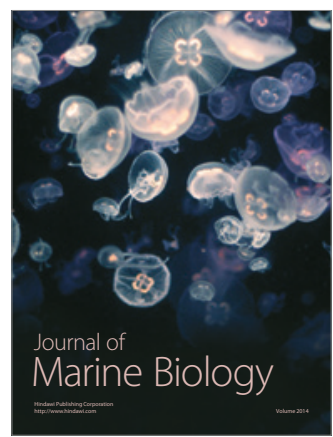

